\title{
Using Semantic Web to Enhance User Understandability for Online Shopping License Agreement
}

\author{
Muhammad Asfand-e-yar and A Min Tjoa \\ Vienna University of Technology, Favoritenstraße 9-11/188-1, Vienna, Austria \\ (asfandeyar, amin) @ifs.tuwien.ac.at
}

\begin{abstract}
Normally, a common user sign license agreement without understanding the agreement. License agreements are a form of information, which describes product's usage and its terms and conditions. Habitually, users agree with it but without understanding. In the today's information age, there is no integration of license agreements with any current technology. The contents of license agreements are out of scope for search engines. Management of license agreements using Semantic Web is a multi-disciplinary challenge, involving categorization of common features and structuring the required information in such semantics that is easily extendable and fulfilling the requirements of common user.

In this paper construction of Semantic Web model for Online Shopping license agreement is discussed. The user requirements facilitate the construction of License Ontological model. Moreover, rules are used to capture the complex statements of "terms and conditions". Finally, an explicit semantic model for agreements is constructed that facilitates users' queries.
\end{abstract}

Keywords: License Agreements, Semantic Web, License Ontological Model, Digital License Agreement.

\section{Introduction}

In general, installing software or using any services requires the user's agreement to terms and conditions. The terms and conditions describe issues such as functionality, restrictions of use, and about other legal acts such as jurisdiction restriction and intellectual property rights. These terms and conditions describe various legal acts of an agreement. The legal acts contained in agreements are often difficult for a user to understand. Therefore, users do not read such lengthy agreements and bypass the agreement by signing, without understanding. In such situations user might commit illegal act unintentionally. Therefore, an easy understandable solution is required in order to facilitate user's requirements. Hence, a solution in form of agreements' repository is provided that is easy for a user to select an appropriate product. Moreover, Semantic model for online shopping license agreements is discussed in this paper. With the help of the Semantic model user queries are answered in conjunction with user's requirements. 
License Agreements: The agreement is a legal binding between two parties and if any violation occurs then defined penalties are applied. Similarly, using web services requires the user's agreement to the terms and conditions. Agreements describe issues related to product's functionality, restrictions on its use, may specify the number of users that can work in a connected environment, state relevant laws if any, rules pertaining to the distribution of the product, modifications that can be made and the payment procedure [1]. In online shopping centers a legal contract occurs between a buyer and seller while purchasing a product.

The concepts contained in agreements are often difficult for a user to understand. Moreover, the terms and conditions are different nearly in every license agreement. Therefore, user agrees with agreement, without deeper understanding, which might lead to a user's inadvertent implication in an illegal act. Approximately every agreement applies penalties, if terms and conditions are violated.

User Awareness: For the awareness and understanding agreements, few efforts are done for example; GPL-violations, it is an awareness project about the violation of GNU licensing agreements. "GPL-violation" is a communication platform between all parties that are involved in licensing of open source software for example authors and copyright holders, vendors and users [2].

Business Software Alliance (BSA) is an IT industry group that helps their member groups in controlling piracy of software and hardware tools. BSA provides awareness and educate people about the ethical and digital security risk associated with use of unlicensed software [3].

The work done by GPL-violations and BSA serves for user awareness; while the Semantic model provides a solution, in selecting a required license according to the needs of a user, and also make awareness of penalties.

\section{Related Work}

In the literature, a plethora of studies has been conducted for developing a Semantic Web model to fulfill user requirements. The research is based on two sections, initially, it summaries fundamental concepts of license agreements, by considering different type of existing licenses. Secondly, it highlights the use of ontology in information retrieval, management, automatic extracting of meta-data and different concepts related to Semantic Web. According to the multifaceted nature of the research work, we will describe the problem from more than one angle.

\subsection{License Agreements}

Organizations do their best to control the misuse of License Agreements. Hence, penalties are defined to assure the legal use, defined in "terms and conditions".

Various license verifying systems have been developed. These systems help to ensure that a licensed version of a product is properly and legally installed on each computer in an organization. Microsoft verifies the installing products online, before completing the installation of the software product on a system [4]. This clarifies that 
the granter of license wants her product to be used legally and according to the terms and conditions defined in the license agreement.

Online shopping agreements are mostly similar to each other and have a lower degree of complexity as software licenses [5]. In these types of agreements, rights of service providers and customers are clearly defined. In some agreements service providers are the sellers and provide a facility of purchasing from Web sites. While other agreements are totally different from service provides, for example in case of eBay. Only two types of online shopping agreements are explained and used as pars pro toto in this paper, i.e. Amazon [6] and eBay [7, 8] license agreements.

\subsection{Semantic Web}

Digital Right Expression Languages and Policy Management are the two areas, covered in this paper. Digital Right Management is an access control technology that is used to impose limitations on usage of digital contents and devices. It controls the illegal use of digital media by preventing access, copy or conversion to other formats by end users. Semantic Web technologies facilitates in creating ontology for copyright agreements. These copyright agreements are digital rights used to implement legal limitation.

\section{Digital Rights Expression Languages}

Rights Expression Languages (REL) reflects licenses legal requirements, by gathering terms and required relations of contents. REL creation is based on an ontology using existing standards for example ODRL, XrML and Creative Commons. Usage of contents depends on the rights of a license provider, granted to licensee. REL with DRM translates licenses and uses ontology to deduce copyrights, context description and different type of expressions used in a license agreement. The REL ontology terms are mapped to a legal dictionary, which consists of legal classifications and legal rules used in the license law [9].

\section{Privacy Policy Matching}

Nyre discusses the issues related to "privacy policy matching" [10]. They suggested, arranging policies of service providers according to customer requirements. Customer requirements are translated to policies using privacy preferences of the provider, for matching the customer requirements with the service provider polices. Privacy preference provider is introduced as an intermediate channel between service provider and customers; to solve, the issue of matching and arranging the policies for service providers.

The word "policy" used in this method is similar as terms and conditions of license agreement. These policies are defined by the service provider, for example in online shopping license agreements. The main problem of this method is to maintain an intermediate channel as "privacy preference provider" for updates of customer requirements. The second problem is to translate customer's requirements to policies, at this intermediate level, and then match these policies to service provider's policies. 
Handling these problems, according to the above method, is worse in case to deal with complex policies of service providers. To solve the above mentioned problem, we propose in this paper a solution using Semantic Web technologies.

\section{Use Case Scenarios}

Use case scenario elaborates user's requirements to achieve specific results. License model is constructed to facilitate a common user. Therefore, each license is categorized similarly. The model facilitates a common user to acquire her required information from a license. The model is designed to easily adopt documented agreement using Semantic technologies. The Semantic Web model of agreements covers all common features of an agreement. A user scenario is discussed below;

\section{Online Shopping}

Using services of online shopping web sites, the policies should be agreeable by both sellers and buyers. Sellers defined their own polices, without conflicting the police of online shopping. In case of conflicting policies, seller has either to face penalties or in worst case could be ban to use the services. This scenario is explained below:

A seller wants to sell her items through online shopping web site. The used items are placed for biding by the seller. One of the buyers received the item, with functionality problem. According to polices of online shopping, a buyer can claim for reimbursement. Therefore, the buyer claimed for reimbursement. In this case, the seller didn't define a proper procedure for reimbursement. The online shopping company paid for the damages, and ceased the seller's account and banded him from selling her item from the site. Such problems normally happens when user bypass the agreement, before using services.

\section{$4 \quad$ Semantic Web and License Agreements}

Semantic Web uses ontology as its basic component and uses taxonomy of information as an expressing knowledge to design ontology. Defining a domain of knowledge for license agreements, a root ontology (named; License Ontology) is created using bottom up approach, as discussed in $[5,11]$.

The ontological structure for license agreements is categorized in two parts, i.e. License Ontology and sub license ontologies. The License Ontology is developed from common features of different license agreements. The License Ontology is extended to sub license ontologies, in order to design ontology as a meaningful license agreement. Sub-license ontology describes a specific license agreement.

The resulting model is equipped with rules according to license agreements. Functionality of entire process depends on search and comparison of user requirements using License Ontology. Query results obtained from sub license ontologies are sent to user for final approval. 


\subsection{Sub License Ontology}

Sub ontology is extracted from parent ontology to elaborate a section of domain knowledge. The extracted sub-ontology has following two features 1) consistency and 2) completeness. Sub-ontology concept is explained by proposing four methods i.e. extend, add, merge and replace. We used extended method of sub-ontology concept. The extend method depends on new features of sub-ontology engineering, which includes concepts, properties, relationships and mapping. These sub license ontologies are categorized into Online Shopping License Ontology and Software License Ontology. The Software License Ontology is explained in detail in [5].

\section{Online Shopping License Ontology}

Construction of Online Shopping License Ontology is based on two different agreements. These include Amazon license agreement and eBay license agreement. These agreements have most common features. Online Shopping License Ontology is an extension of License Ontology, as shown in Fig.1. The Online Shopping License Ontology extends all the features of License Ontology. A class named "Reimbursement" is added to Online Shopping License Ontology. The class is required to provide a concept of reimbursement method for customer, after purchasing product. Required properties are added to define the relationship of reimbursement class with customer and seller classes in the Online Shopping License Ontology.

Online Shopping License Ontology has defined individuals. These defined individuals are connected with each other, according to agreements, to define complete meaning of License Ontology

\section{$5 \quad$ Rules Used for Constructing License Ontology}

Rules are needed for inference about classes and properties, mapping ontologies, transforming data in different format, using complex queries, axioms and many more. The aim is to populate the domain of License Ontology by means of rules, created form analyzing set of text documents. The entities are used in License Ontology and sub license ontologies on basis of terms and condition of agreements. Rule applied on Online Shopping License Ontology is described as follows;

\section{Disjoint Classes in Ontology}

In License Ontology only those classes that have information related to terms and conditions of agreement are considered disjoint. In License Ontology, classes used under "Agreement Items" class, i.e. "Breach", "Core Agreement" and "Non Core Agreement", and also the sub-classes of these defined classes are disjoint to each other. 


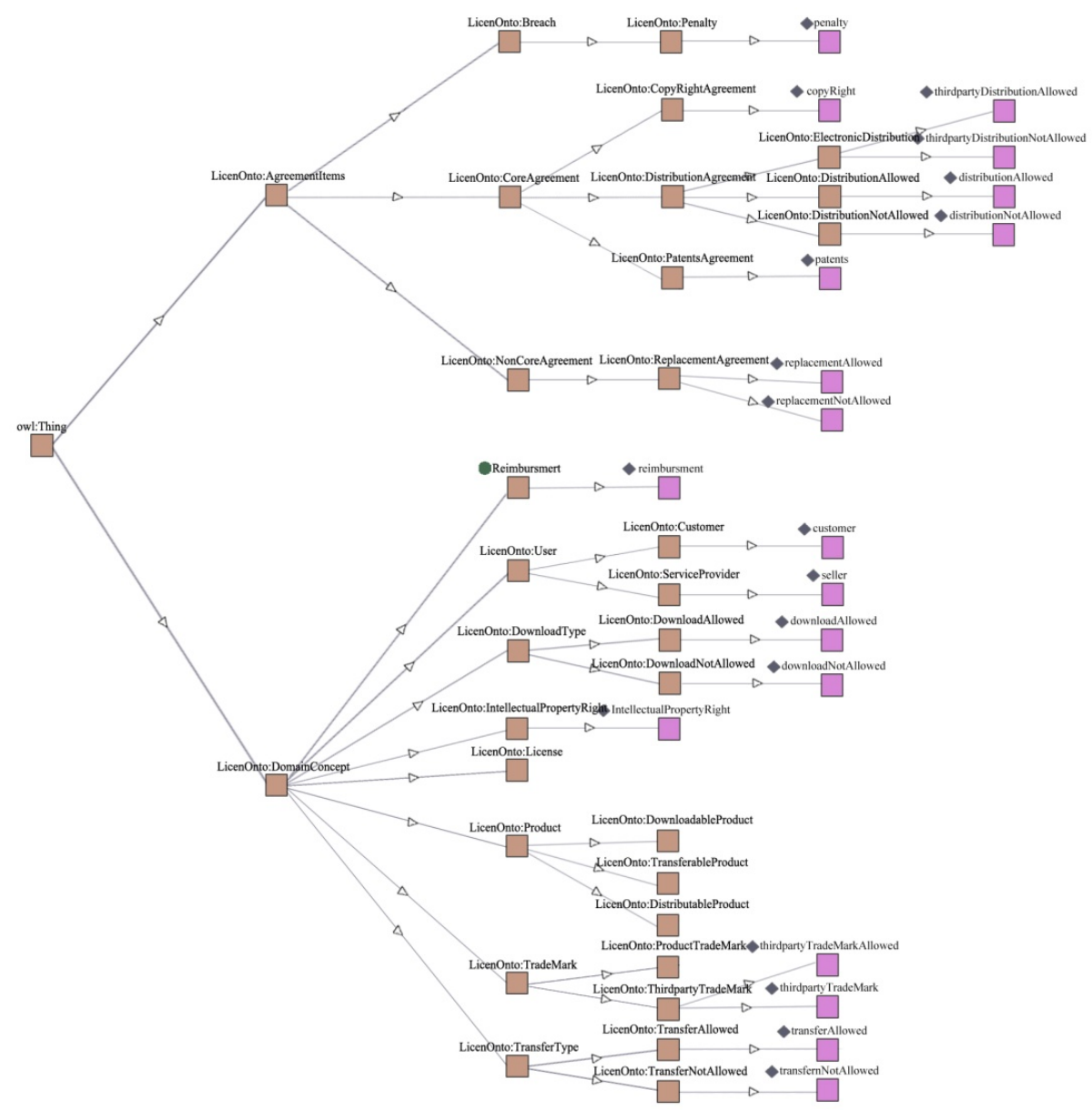

Fig. 1. Online Shopping License Ontology

\section{Restriction Used for Product Class}

Details about product's legal usage are documented by license agreement i.e. reflected as below;

$$
\text { Product } \sqsubseteq \forall \text { isDocumentedBy . License }
$$

It states that for all values of product should have an agreement (i.e. "License"). Vice verse the restriction applied also on "License" class because both properties are inverse to each other;

$$
\text { isDocumented } \equiv \text { documents }{ }^{-}
$$




\section{$6 \quad$ Reasoning License Ontology}

Acquiring information from ontology can be determined by rules. After having information from ontology by applying rules, then the next step is to represent the information. In this section, ten rules are introduced that are built according to previously discussed user scenarios. These rules describe complete requirements of a user, to search for appropriate license using ontologies.

\section{Online Payment}

Rule for online payment provides information, about procedure of payment according to an agreement. The rule describes, that a product have ontology. And according to the ontology, it is inquired that payment procedure supports online payment of the product or not. Then availability of user permissions is checked, for online purchasing of the product, according to the ontology. Thus, according to this rule, an end user is allowed to purchase the product according to an agreement as described below;

(?product LicenOnto:hasLicense ?license) (?license LicenOnto:supportPaymentMethod LicenOnto:Online) (?user profile:paymentMethod profile:Online) $\longrightarrow$ (?user profile:canPayOnline ?product)

\section{Method of Receiving a Product}

The following rule is about downloading a product according to ontology. It states that a product having agreement and allows a user to download the product. The rule examines whether a user can download the product or not. According to ontology, if antecedent part holds answer then consequent part of the rule will also hold answer and allow user to download the product online. The product could be e-book, etc.

(?product LicenOnto:hasA ?license) (?license LicenOnto:receiveMethod SoftOnto:Download) (?user profile:receiveMethod profile:Online) $\longrightarrow$

(?user SoftOnto:canDownload ?product)

\section{Replacing of Damaged Product}

"Replacing of Damaged Product" rule is about replacing a product, if the product is damage before receiving. Therefore, according to ontology the availability of replacing damaged product is evaluated against respective agreement. If a method of replacing a damaged product is allowed according to ontology then the product is allowed for replacement. According to this rule, the possibility of replacing the damaged product is determined according to agreement.

(?user profile:ownsProduct ?product) (?product LicenOnto:hasLicense ?license) (?license LicenOnto:replaceMethod SoftOnto:Return) $\longrightarrow$

(?user SoftOnto:canReturn ?product) 


\section{Reimbursement of Payment}

Rule of reimbursement describes the method of reimbursement of expenses after identifying fault in a received product. In agreement normally the reimbursement procedure is defined for customers. Therefore, in ontology a rule is used to inform customer about availability of reimbursement procedure according to an agreement. Whenever, such situation occurs then following rule is used to find out the availability of reimbursement method, according to agreement.

(?user profile:hasPurchased ?product) (?product LicenOnto:hasLicense ?license) (?license LicenOnto:replaceMethod OnliShopOnto:reimbursement) $\longrightarrow$

(?user OnliShopOnto:canReturn OnliShopOnto:reimbursement)

\section{Conclusion}

Initially, undertaking an agreement without understanding is a major issue. Habitually customers agree with terms and conditions of an agreement without understanding. Incomplete knowledge and understanding about a license agreement in general and agree with an agreement without understanding specifically - is a main problem for one's own self. To solve the issue, a License Ontology is constructed, which is not only a platform for different license agreements to reside but users can access their required information by querying ontology. The application is designed in such a way that different license agreements can be easily plotted on same platform with minor changes according to agreement.

Based on ontologies, Digital License Agreement application (user interface of License Ontology) uses triples to define a meaningful sentence. Therefore, the results are based on the triples. These triples are defined so that the meaning of a statement in a license agreement should not be changed and the triples should be meaningful according to common user understanding.

\section{Future Work}

The Semantic Web model for license agreements is extendable, to adopt other license agreements, for example agreements of service providing companies, hardware products, etc. Following agreements could also be model using the License Ontology.

\section{Cross Boundary Agreements and its Conflicts}

"Airline's Terms and Condition" could be constructed with similar method, as used for previous explained sub ontologies. Whenever, centralization of same categories of license agreements are achieved, then the ontology will be able to find out the conflicts between different laws (i.e. terms and conditions or license agreements). For example, airlines have to make agreements to use airports and airspace of other territories. Normally, settling downing the conflicts in agreements, consumes maximum time. Such system will not only explain the terms and conditions of each partner but 
will also explain the compromising statements that took place between them. These compromising statements will be helpful for upcoming organization to settle down their conflicts with the same/other organization.

\section{SLAs and Business Processes}

Cloud computing is a blend of concepts evolved from the SOA, grid computing and Virtualization. Adapting to this new way of computing is a hindrance for the companies to meet their requirements [12]. Laws and policies issues that must be addressed while adopting cloud computing for particular jurisdiction. Some of the challenging issues are; access, reliability, security, liability, IPR, ownership, portability and auditability. The failure of these issues causes resistance to provide services. Lingering mistrust has a negative impact on service providers. Cloud providers also face jurisdictional issues, such as: government intervention and costs of doing business [13].

We propose a Semantic solution for SLA cloud services which supports the consumers in finding the appropriate services that matches their specific requirements. As well as with awareness, consumers have choices to select the proper service according to their requirements. The proposed approach aims at globally unified SLA ontology. Organizations using this approach are believed to be benefited as (i) the categorization of agreements helps to define their "terms and conditions" using Semantic Web technology accordingly (ii) easy in understanding and comparing the different agreements for choosing a better option.

Mapping ontology will be used to relate business process and functions to cloud computing. Semantic rules encapsulate the tacit knowledge of enterprise business. The rules and relations used in Semantic Web technology facilitates businesses in selecting proper service of cloud computing. The selection of services depends on the requirements of customers according to business processes and functions.

\section{References}

1. Alliance, B.S.: Why a License matters? (2012),

http://ww2 .bsa.org/country/Anti-

Piracy/WhyaLicenseMatters.aspx

2. Welte, H.: About the gpl-violations.org project (2010), http: / /www.gpl-violations.org/

3. BSA. About BSA \& Members (2012), http: / /ww2 . bsa.org/country/BSAandMembers . aspx

4. Microsoft. Genuine Windows: FAQ (2012), http: / /windows.microsoft. com/en-US/windows/help/genuine/faq

5. Asfandeyar, M., Anjomshoaa, A., Weippl, E.R., Tjoa, A.M.: Exploiting ontology for software license agreements. International Journal of Software and Informatics (IJSI) 4, 89-100 (2010)

6. Services, A.W. AWS Customer Agreement (2012),

http: / /aws . amazon.com/de/agreement/

7. eBay. Your User Agreement (2012),

http://pages.ebay.ie/help/policies/user-agreement.html 
8. Post, E.T. License Agreement-eBay Trading Post (2012), http: / /pages.ebay.ie/tradingassistants / license-agreement.html

9. Nadah, N., Rosnay, M.D., Bachimont, B.: License Digital Content With a Generic Ontology Escaping From The Jungle of Rights Expression Languages. In: International Conference on Hybrid Information Technology, pp. 65-69. ACM (2007)

10. Nyre, A.A., Bernsmed, K., Solvar, B., Pedersen, S.: A Server-Side Approach to Privacy Policy Matching. In: 1st International Workshop on Privacy by design (PDB), ARES 2011 (2011)

11. Asfand-e-yar, M., Anjomshoaa, A., Weippl, E.R., Tjoa, A Min: Blending the Sketched Use Case Scenario with License Agreements Using Semantics. In: Karagiannis, D., Jin, Z. (eds.) KSEM 2009. LNCS, vol. 5914, pp. 275-284. Springer, Heidelberg (2009)

12. Anjomshoaa, A., Tjoa, A Min: How the Cloud Computing Paradigm Could Shape the Future of Enterprise Information Processing. In: Proceedings of 13th International Conference on Information Integration and Web-based Applications Services (iiWAS). IEEE, Ho Chi Minh City (2011)

13. Jaeger Paul, T., Lin, J., Justin, M.G., Shannon, N.S.: Where is the cloud? Geography, economics, environment and jurisdiction in cloud computing. 1st Monday, Peer Reviewed Journal on the Internet 14(5) (2009) 